\title{
Primary Duodenal Adenocarcinoma: About Two Cases
}

\author{
Asmaa Elkarouachi, Ahmed Ballati, Asmar Mohamed, Saâd Rifki Jai, Driss Erguibi, Rachid Boufettal, \\ and Farid Chehab
}

\begin{abstract}
This article focuses on the symptomatic and diagnostic problems of primary duodenal adenocarcinoma by way of two case reports and a literature review.The diagnosis of primary duodenal adenocarcinoma is often delayed because its symptoms may be absent until the tumor has progressed, thus leading to a delay of several months. Patients typically present with a long history of variable and vague symptoms, and many are diagnosed with advanced disease. As regards clinical manifestations, abdominal pain is the most frequent symptom. The majority of these tumors are found to have infiltrated the duodenal wall at presentation, with many being unresectable due to local and distal invasion.

Index Terms - adenocarcinoma; duodenum; surgery.
\end{abstract}

\section{INTRODUCTION}

Primary adenocarcinoma of the duodenum is a rare tumor and represents $50 \%$ of adenocarcinoma of the small intestine Because of its non-specific clinical signs, it poses a problem of delayed diagnosis and management, its treatment is mainly based on surgery. We report the observations of two patients admitted with duodenal adenocarcinoma requiring radical surgical treatment for one and palliative treatment for the other.

\section{PATIENTS AND OBSERVATIONS}

\section{A. $\quad$ Case 1}

The patient was 61 years old with no particular pathological history. One and a half months before admission, he presented progressive epigastralgia , relieved by late post-prandial food vomiting and melenas of low abundance without jaundice or transit disorders. The symptomatology evolved in a context of apyrexia and alteration of the general state .The clinical examination found a apyretic patient with a blood pressure is $130 / 70 \mathrm{~mm}$ $\mathrm{Hg}$ and a pulse rate of 60 beats/minute, a body mass index of $25 \mathrm{~kg} / \mathrm{m} 2$ without jaundice. The abdominal examination showed a normal abdomen without hepatomegaly, splenomegaly or palpable mass the digital rectal examination showed no abnormalities the rest of the somatic examination .Liver and kidney function tests were normal .The tumor markers ACE and CA 19.9 were normal. Oesogastroduodenal fibroscopy revealed a budding tumor aspect of the second duodenum. Histological examination revealed the presence of a moderately differentiated invasive Lieberkuhnian adenocarcinoma.

Abdominal ultrasound was normal, chest x-ray was normal.
The abdominal-pelvic CT scan showed parietal thickening (Figure 1) of the lower second duodenum in theabove the papilla infiltrated by a heterogeneous process of $28 \times 23$ millimeters invaded the lower part of the head of the pancreas without invasion of the peridodenal fat with normal liver and commun bile duct. MRI had objectified the presence of a tissue process of the third duodenum measuring 25 millimeters in diameter of a heterogeneous aspet. The patient's file was discussed in a multidisciplinary concentration meeting or a surgical intervention was decided. The preoperative assessment was normal apart from the anaemia. A median laparotomy was performed under general anaesthesia. Surgical exploration had shown a tumour of the second and third duodenum of about 4 to 5 centimeters long axis invading the head of the pancreas intimately without hepatic metastases or ascites. In front of this localized aspect of the tumor, the patient had benefited from a cephalic duodenopancreatectomy with a restoration of pancreaticobiliodigestive continuity according to Child's set-up. The immediate postoperative follow-up was normal. Anatomopathological analysis of the surgical specimen showed a well-differentiated and invasive duodenal pan parietal adenocarcinoma measuring $6 \mathrm{~cm}$ long axis extending to the duodenal papilla infiltrating the pancreas with perinerve fattening and presence of vascular emboli without lymph node metastases the tumour was classified as pT4N0.The patient had received four courses of chemotherapy based on 5 fluorouracil oxaplatine and capecitabine (XELOX) with good tolerance. The follow-up abdominal-pelvic CT scan showed no suspicious duodenal thickening or secondary lesions. The patient is still alive the current decline is 10 months.

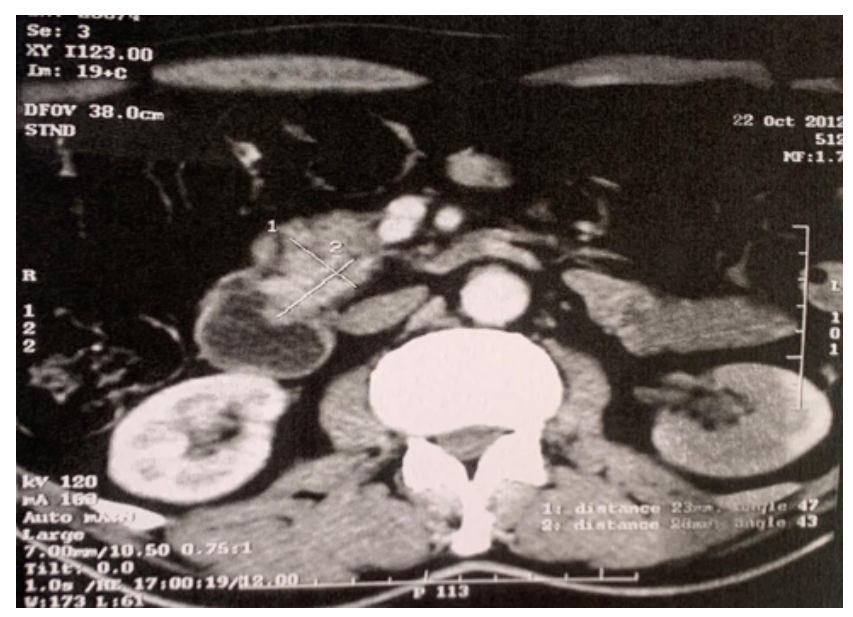

Fig 1: Parietal thickening of the second duodenum 


\section{B. Case 2}

The second patient was 37 years old his father had died 13 years earlier of a metastatic gastric embolism tumour. Three months prior to his hospitalisation, he presented with late postprandial bilious vomiting associated without jaundice or digestive haemorrhage. This symptomatology evolved in a context of unquantified fever and alteration of general state with anorexia, asthenia and weight loss of 22 kilograms in three months. The clinical examination on admission had found a conscious patient with apyretic and eupnea. His blood pressure was $120 / 70 \mathrm{~mm} \mathrm{Hg}$, and his pulse 88 beats/minute. His body mass index was $24 \mathrm{Kg} / \mathrm{m} 2$. He had conjunctival pallor without mucocutaneous jaundice or edema of the lower limbs. The abdominal examination had found a painless, supple abdomen that was mobile on breathing with no palpable mass, hepatosplenomegaly or dullness of the flanks; the digital rectal examination found no abnormalities. The rest of the somatic examination was unremarkable. The Esogastroduodenal fibroscopy revealed a friable and haemorrhagic tumour proliferation in the $3 \mathrm{rd}$ portion of the duodenum. The Histological examination showed a poorly differentiated and infiltrating adenocarcinoma, and oesogastroduodenal transit showed a tight stenosis of the third portion of the duodenum with an irregular and eccentric appearance containing a small lacunae image responsible for retrodilatation of the upstream segment. Abdominal ultrasonography found no abnormalities. The Chest X-ray was normal. The abdominal pelvic CT scan had shown a tumor process measuring $24 \mathrm{X}$ 34 milimeters at the level of the third duodenum pushing the head of the pancreas without detectable ductal dilatation and it infiltrated the inferior vena cava the superior mesenteric vein which is most probably thrombosed without detectable deep adenopathies. At the end of this assessment the patient was proposed for surgery. The preoperative assessment was normal apart from anaemia. Surgical exploration found a tumour of the third fixed duodenum invading the upper mesenteric asterior and the gastrocolic trunk of Henle.

The patient underwent gastrojejunal anastomosis with subhepatic drainage by Redon's drain. The immediate postoperative follow-up was simple. The patient was declared discharged at D6 postoperatively. The patient had received three courses of chemotherapy (FOLFOX) with almost stabilization of the tumor mass. The abdominal pelvic CT scan performed after chemotherapy showed the stable appearance of the tumour mass, a change of protocol was decided and the patient was given three courses of chemotherapy (FOLFIRI). The thoraco-abdominopelvic CT scan done after the three chemotherapy cures had shown the progression of the duodenal tumour process measuring 5.7 X 6.9 centimeters in diameter and a stomach of stasis associated with fat infiltration with the presence of mesenteric adenopathies. After the third treatment, the patient presented a right hypochondrium pain associated with a cutaneous mucous jaundice with dark urine and discoloured stools, all evolving in a context of fever and alteration of general state. A biological assessment was carried out and showed hepatic cytolysis and a cholestasis syndrome. Abdominal ultrasound showed dilatation of the main bile duct $(12 \mathrm{~mm})$ with discrete dilatation of the intrahepatic bile ducts without any individualizable obstacle. A surgical reoperation was decided in a multidisciplinary concentration meeting but was not carried out due to the deterioration of the patient's general condition. The patient had died at home; the delay was 14 months.

\section{DISCUSSION}

The duodenal adenocarcinoma accounts for about $0.35 \%$ of gastrointestinal cancers, it is a rare tumor first described by Hamberger in 1746 [2,3]. The mean age is 67 years with a slight predominance for the male sex [4].The patients do not usually present until the tumors have reached a size sufficient to cause symptoms, they are not specific and include : abdominal pain, nausea, vomiting, fatigue, weakness and weight loss. Anemia, gastrointestinal obstruction and jaundice are symptoms associated with advanced disease. Abdominal pain is the most frequent symptom, associated with $56 \%$ of cases [5]. The clinical examination depends on the location and size of the tumour and its degree of extension: it can be strictly normal or find an epigastric mass with jaundice and alteration of the general condition. The positive diagnosis of duodenal adenocarcinoma is based on endoscopy because it allows simultaneous visualization and biopsy. The Duodenal adenocarcinomas are localized in the second duodenum in $59.2 \%$ of cases, in the first duodenum in $15 \%$ of cases and in the third or fourth duodenum in $45 \%$ of cases [6].An endoscopic ultrasound can be performed simultaneously to evaluate local extension or lymphadenopathy. The thoracoabdomino-pelvic CT scan makes it possible to study the tumour, the relationships and the locoregional and remote extension [2]. Resection of the primary tumour is the only curative treatment for duodenal adenocarcinoma Pancreaticoduodenectomy is often performed for tumours of the second portion of the duodenum and for proximal and distal infiltrating duodenal tumours to ensure radical resection with adequate lymph node healing [7] .A segmental duodenal resection is possible for proximal (first portion of the duodenum) or distal (third portion of the duodenum) tumours, on the left side of the superior mesenteric artery), or in case of a tumour of the duodenojejunal angle[4]. In case of an unresectable tumour, a gastroenterostomy or placement of a duodenal prosthesis in case of symptomatic stenosis. A biliodigestive bypass will be offered endoscopically or by surgery in case of biliary tract invasion if biliary prosthesis cannot be inserted[2]. The Oxaliplatin is generally offered to high-risk patients, such as those with positive lymph nodes. In some series, adjuvant radiotherapy is associated with better local control but no difference in overall survival[5]. Despite advances in resuscitation, the mortality of pancreaduodenectomy is $5 \%$ in expert centers with morbidity of $15-40 \%$

\section{CONCLUSION}

The duodenal adenocarcinoma is a rare but aggressive malignancy. Because of the non-specific symptoms it presents and the difficulty in confirming a diagnosis. Nevertheless, aggressive surgical resection, when possible, offers the best chance of survival. 


\section{REFERENCES}

[1] Ecker BL, McMillan MT, Datta J, Mamtani R, Giantonio BJ, Dempsey DT, Fraker DL, Drebin JA, Karakousis GC, Roses RE. Efficacy of adjuvant chemotherapy for small bowel adenocarcinoma: A propensity score-matched analysis.Cancer. 2016 Mar 1; 122(5):693-70.

[2] KRAMI, H., BENZZOUBEIR, N., OUAZZANI, L., et al. Primitive adenocarcinomas of the duodenum. Med Maghreb, 1997, vol. 63, pp. 29-32.

[3] ALWMARK, ANDERS, ANDERSSON, A., et LASSON, A. R. K. E. Primary carcinoma of the duodenum. Annals of surgery, 1980, vol. 191, no 1, p. 13.

[4] ZAANAN, A., AFCHAIN, P., CARRERE, N., et al. Adenocarcinoma of the small intestine. Clinical and Biological Gastroenterology, 2010, vol. 34 (6-7): 371-379.

[5] CLOYD, Jordan M., GEORGE, Elizabeth, et VISSER, Brendan C. Duodenal adenocarcinoma: Advances in diagnosis and surgical management. World journal of gastrointestinal surgery, 2016, vol. 8, no 3, p. 212.

[6] MARKOGIANNAKIS, Haridimos, THEODOROU, Dimitrios, TOUTOUZAS, Konstantinos G., et al. Adenocarcinoma of the third and fourth portion of the duodenum: a case report and review of the literature. Cases journal, 2008, vol. 1, no 1, p. 98.

[7] MEIJER, Laura L., ALBERGA, Anna J., DE BAKKER, Jacob K., et al. Outcomes and treatment options for duodenal adenocarcinoma: a systematic review and meta-analysis. Annals of surgical oncology, 2018, vol. 25, no 9, p. 2681-2692.

[8] LÉPILLIEZ, Vincent, NAPOLÉON, Bertrand, PONCHON, Thierry, et al. Duodenal adenomas: diagnostic strategy and therapeutic modalities. Clinical and Biological Gastroenterology, 2009, vol. 33 (4): 240-246.

\section{Asma Elkarouachi}

20240, Casablanca,Morocco

Telephone :00212614110017

e-mail:dr.elkarouachi@gmail.com

Institution: Department of General Surgery III, Ibn Rochd University Hospital, Casablanca, Morocco.

\section{Ahmed Ballati}

e-mail:ahmedballati@gmail.com

Institution: Department of General Surgery III, Ibn Rochd University Hospital, Casablanca, Morocco.

\section{Mohamed Asmar}

e-mail:asmar.med87@gmail.com

Institution: Department of General Surgery III, Ibn Rochd University Hospital, Casablanca, Morocco.

\section{Saad Rifki El Jai}

e-mail: Saadjai@yahoo.fr

Institution: Department of General Surgery III, Ibn Rochd University Hospital, Casablanca, Morocco.

\section{Rachid Boufettal}

e-mail: racbouf@hotmail.fr

Institution: Department of General Surgery III, Ibn Rochd University Hospital, Casablanca, Morocco.

\section{Driss Errguibi}

e-mail: erguibi_d@yahoo.fr

Institution: Department of General Surgery III, Ibn Rochd University

Hospital, Casablanca, Morocco.

\section{Farid Chehab}

e-mail: Farid_chehab@yahoo.fr

Head of the digestive cancer and liver transplant surgery department

Institution: Department of General Surgery III, Ibn Rochd University

Hospital, Casablanca, Morocco. 